Communication

\title{
New Methodology for the Synthesis of Thiobarbiturates Mediated by Manganese(III) Acetate
}

\author{
Ahlem Bouhlel, Christophe Curti and Patrice Vanelle * \\ Laboratoire de Pharmaco-Chimie Radicalaire, Faculté de Pharmacie, Institut de Chimie Radicalaire \\ ICR, UMR 7273, Aix-Marseille Univ, CNRS, 27 Bd Jean Moulin, CS 30064, \\ 13385 Marseille Cedex 05, France \\ * Author to whom correspondence should be addressed; E-Mail: patrice.vanelle@univ-amu.fr; \\ Tel.: +33-491-835-580; Fax: +33-491-794-677.
}

Received: 15 March 2012; in revised form: 30 March 2012 / Accepted: 31 March 2012 /

Published: 10 April 2012

\begin{abstract}
A three step synthesis of various thiobarbiturate derivatives 17-24 was established. The first step is mediated by $\mathrm{Mn}(\mathrm{OAc})_{3}$, in order to generate a carbon-carbon bond between a terminal alkene and malonate. Derivatives 1-8 were obtained in moderate to good yields under mild conditions. This key step allows synthesis of a wide variety of lipophilic thiobarbiturates, which could be tested for their anticonvulsive or anesthesic potential.
\end{abstract}

Keywords: manganese(III) acetate; barbiturates; radical

\section{Introduction}

Manganese(III) acetate has been extensively explored during the past decades, and it remains an useful tool for carbon-carbon bond formation [1,2]. Its specificity to carbonyl derivatives allows a wide variety of radical synthetic applications, as studied on acetoacetate [3], $\beta$-ketoesters [4], $\beta$-ketonitriles [5,6] and $\beta$-ketosulfones [7-9]. Malonate derivatives, key-step substrates for barbiturates synthesis [10,11], are also useful substrates for manganese(III) acetate-mediated reactions [12,13]. In continuation of our research program centered on the design and synthesis of original molecules with pharmacological properties [14-18], we propose herein a manganese(III) acetate-mediated multistep synthesis of new original barbiturates. 
Barbiturate derivatives are a well-known pharmacological class with anticonvulsive, sedative and anesthetic properties [19]. Original barbiturates were also recently reported as matrix metalloproteinase inhibitors with potent pharmacological applications against focal cerebral ischemia after acute stroke [20] and cancer cells invasiveness inhibitors [21]. Barbiturate derivatives also show antitubercular [22], PPAR- $\gamma$ agonist [23-25] and protein kinase $C$ inhibitor [26] activities.

The lipophilicity of barbiturates is an important parameter which enhances anesthetic onset [27]. It can be improved by replacing oxygen by a sulfur [28], as seen with the very short acting barbiturate thiopenthal. Substituents on the carbons of the barbituric acid scaffold also have a great influence on the pharmacological activity [27,29]. Our methodology allows synthesis of a wide variety of substituted barbiturates, which could be tested for their anticonvulsive or anesthetic potentialities.

\section{Results and Discussion}

Starting from malonate barbiturate precursors, reproducible methodology for synthesis of various and highly functionalized derivatives was established. As reported in previously described mechanisms [30], $\mathrm{Mn}(\mathrm{OAc})_{3}$ and malonates in acetic acid form a $\mathrm{Mn}^{3+}$-enolate complex. $\mathrm{Mn}^{3+}$ is reduced in $\mathrm{Mn}^{2+}$, generating a carbon centered radical between carbonyl groups. This radical reacts with terminal alkene, generating a carbon-carbon bond.

Depending on the malonate substituent, several reactions may occur and in order to investigate a larger variety of barbiturate synthesis possibilities, we have studied three of them. Results are reported in Scheme 1.

Scheme 1. $\mathrm{Mn}(\mathrm{OAc})_{3}$ reactivity towards various malonate derivatives.<smiles>CCOC(=O)C(Cc1ccccc1)C(=O)OCC</smiles><smiles>[R]CC(=C)CC</smiles>
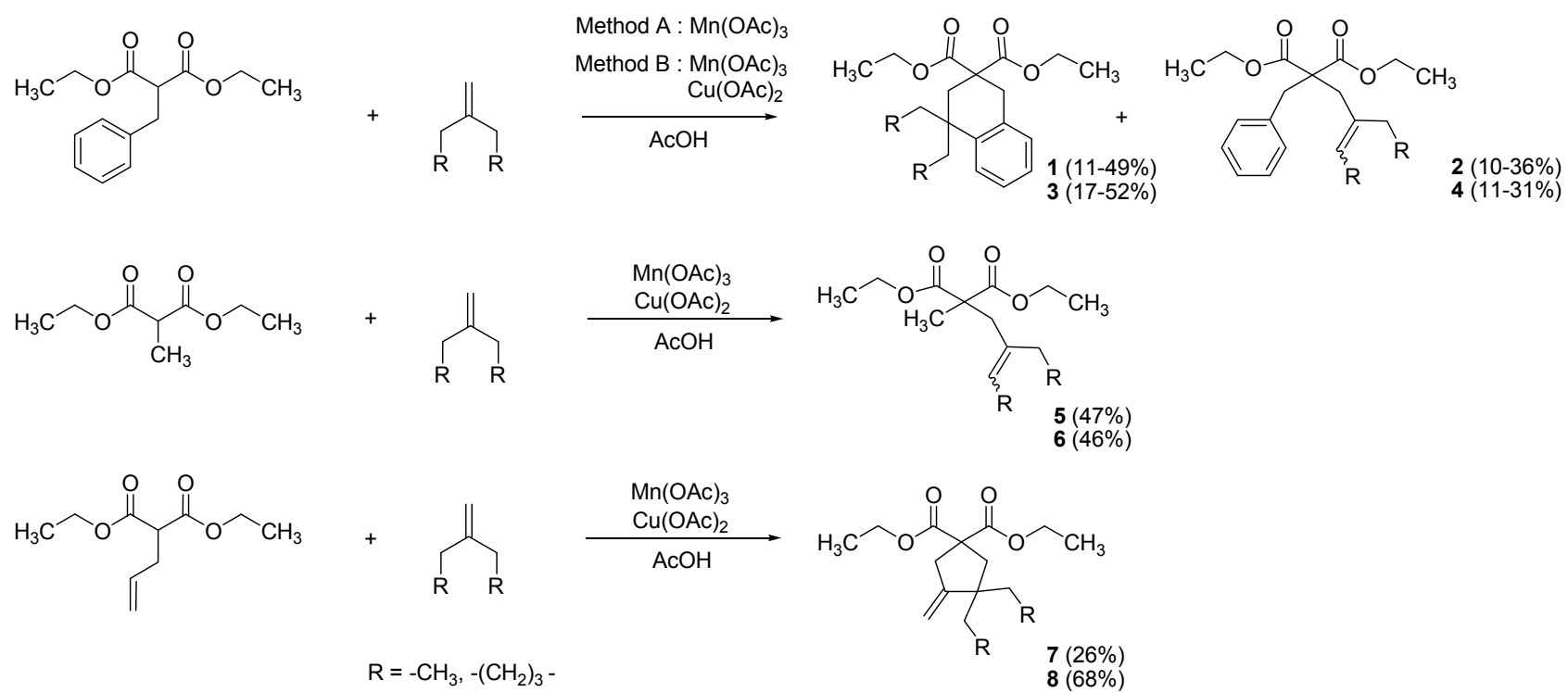

As reported by Citterio and coworkers [31-33], benzylmalonate allowed synthesis of two derivatives: Tetralines 1,3 from radical aromatic substitution, and elimination products $\mathbf{2 , 4}$. We have previously reported different methods for optimizing yields of these two products [34]. For conditions favoring spirocyclic tetralin $\mathbf{1 , 3}$ formation, we divided up the $\mathrm{Mn}(\mathrm{OAc})_{3}$ to ensure moderate oxidizing conditions (method $A$ ). Tetralins 1,3 were obtained as the major compound (49-52\%) and alkenes $\mathbf{2 , 4}$ were observed as secondary products $(10-11 \%)$. Stronger oxidative conditions $\left[\mathrm{Cu}(\mathrm{OAc})_{2}+\mathrm{Mn}(\mathrm{OAc})_{3}\right.$, 
method $B$ ] afforded an increase in elimination products 2,4 (31-36\%), while these conditions drastically decreased yields of tetralines 1,3 (11-17\%).

With methyl malonate, only elimination products 5-6 were obtained with moderate yields (46-47\%). With allyl malonate, cyclization generates a cyclopentane ring [35], and annulation products 7-8 were synthesized (26-68\%). These three different reactivities depend on the malonate substituents, and allow access to a wide variety of substituted substrates for barbiturate synthesis.

C-Functionalized malonates 1-8 thus obtained reacted with thiourea [36], forming thiobarbituric scaffolds 9-16 in moderate to good yields (46-90\%). Results are summarized in Scheme 2 and Table 1.

Scheme 2. Thiobarbituric acid synthesis from malonates 1-8.<smiles>[R]C([R2])(C(=O)OCC)C(=O)OCC</smiles>

1-8<smiles>[13CH3]C(N)=S</smiles>

tBuOK<smiles>[R2]C1([R2])C(=O)NC(=S)NC1=O</smiles>

$9-16(46-90 \%)$

Table 1. Thiobarbituric acids 9-16 synthesis from malonates 1-8.

Entry


Table 1. Cont.

\begin{tabular}{l|c|c|c}
\hline Entry & $\mathbf{R 1 , R 2}$ (malonate) & Product & Yields \\
\hline 6 & &
\end{tabular}

Finally, in order to synthesize intravenous administrable thiobarbiturates, each thiobarbituric acid was turned into the corresponding salt with potassium hydroxide in isopropanol [37], as reported in Scheme 3 .

Scheme 3. Thiobarbituric acid to thiobarbiturate salt formation.<smiles>[R2]C1([R2])C(=O)NC(=S)NC1=O</smiles>

$9-16$

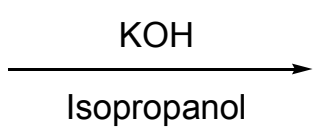

Isopropanol<smiles>[R2]C1([R2])C(=O)N=C([As])NC1=O</smiles>

17-24

\section{Experimental}

\subsection{General}

Microwave-assisted reactions were performed in a multimode microwave oven (ETHOS Synth Lab Station, Ethos start, Milestone Inc., Shelton, CT, USA). Melting points were determined with a B-540 Büchi melting point apparatus. ${ }^{1} \mathrm{H}-\mathrm{NMR}(200 \mathrm{MHz})$ and ${ }^{13} \mathrm{C}-\mathrm{NMR}(50 \mathrm{MHz})$ spectra were recorded on a Bruker ARX 200 spectrometer in $\mathrm{CDCl}_{3}$ or $\mathrm{D}_{2} \mathrm{O}$ at the Service interuniversitaire de RMN de la Faculté de Pharmacie de Marseille. The ${ }^{1} \mathrm{H}-\mathrm{NMR}$ chemical shifts are reported as parts per million downfield from tetramethylsilane $\left(\mathrm{Me}_{4} \mathrm{Si}\right)$, and the ${ }^{13} \mathrm{C}-\mathrm{NMR}$ chemical shifts were referenced to the solvent peaks: $\mathrm{CDCl}_{3}(76.9 \mathrm{ppm})$ or DMSO- $d_{6}(39.6 \mathrm{ppm})$. Absorptions are reported with the following notations: s, singlet; bs, broad singlet; $d$, doublet; t, triplet; q, quartet; $\mathrm{m}$, a more complex multiplet or overlapping multiplets. Elemental analysis and mass spectra which were run on an API-QqToF mass spectrometer were carried out at the Spectropole de la Faculté des Sciences Saint-Jérôme site. Silica gel 60 (Merck, particle size 0.040-0.063 nm, 70-230 mesh ASTM) was used 
for flash column chromatography. TLC were performed on $5 \mathrm{~cm} \times 10 \mathrm{~cm}$ aluminium plates coated with silica gel 60 F-254 (Merck, Gernsteim, Germany) in an appropriate solvent.

\subsection{General Procedure for the Synthesis of Substituted Malonates 1-8}

Method A: A solution of manganese(III) acetate dihydrate (1.68 mmol, $0.45 \mathrm{~g})$ in glacial acetic acid $(55 \mathrm{~mL})$ was heated under microwave irradiation $\left(200 \mathrm{~W}, 80^{\circ} \mathrm{C}\right)$ for $15 \mathrm{~min}$, until dissolution. Then, the reaction mixture was cooled down to $60{ }^{\circ} \mathrm{C}$, and a solution of malonate (3.99 mmol, 1 equiv.) and alkene (11.97 mmol, 3 equiv.) in glacial acetic acid $(5 \mathrm{~mL})$ was added. The mixture was heated under microwave irradiation $\left(200 \mathrm{~W}, 80^{\circ} \mathrm{C}\right)$ for $20 \mathrm{~min}$. Then, the reaction mixture was cooled down to $60{ }^{\circ} \mathrm{C}$ once more, and a second portion of manganese(III) acetate dihydrate ( $1.68 \mathrm{mmol}, 0.45 \mathrm{~g})$ was added. The mixture was heated under microwave irradiation $\left(200 \mathrm{~W}, 80^{\circ} \mathrm{C}\right)$ for $20 \mathrm{~min}$. The addition of manganese(III) acetate dihydrate $(1.68 \mathrm{mmol}, 0.45 \mathrm{~g})$ was repeated three times under the same conditions every $20 \mathrm{~min}$. successively. The reaction mixture was poured into cold water $(100 \mathrm{~mL})$, and extracted with chloroform $(3 \times 70 \mathrm{~mL})$. The organic extracts were collected, washed with saturated aqueous $\mathrm{NaHCO}_{3}(3 \times 50 \mathrm{~mL})$ and brine $(3 \times 50 \mathrm{~mL})$, dried over $\mathrm{MgSO}_{4}$, filtrated, and concentrated under vacuum. The crude product was purified by silica gel chromatography with ethyl acetate/petroleum ether (0.5/9.5) to give corresponding compounds $\mathbf{1}-\mathbf{4}$.

Method B: A solution of manganese(III) acetate dihydrate ( $8.38 \mathrm{mmol}, 2.24 \mathrm{~g}, 2.1 \mathrm{equiv}$.) and copper(II) acetate monohydrate (3.99 mmol, $0.80 \mathrm{~g}, 1$ equiv.) in glacial acetic acid (55 $\mathrm{mL})$ was heated under microwave irradiation $\left(200 \mathrm{~W}, 80^{\circ} \mathrm{C}\right)$ for $15 \mathrm{~min}$, until dissolution. Then, the reaction mixture was cooled down to $60{ }^{\circ} \mathrm{C}$, and a solution of malonate (3.99 mmol, 1 equiv.) and alkene (7.98 mmol, 3 equiv.) in glacial acetic acid $(5 \mathrm{~mL})$ was added. The mixture was heated under microwave irradiation $\left(200 \mathrm{~W}, 80^{\circ} \mathrm{C}\right.$ ) for $60 \mathrm{~min}$. The reaction mixture was poured into cold water $(100 \mathrm{~mL})$, and extracted with chloroform $(3 \times 70 \mathrm{~mL})$. The organic extracts were collected, washed with saturated aqueous $\mathrm{NaHCO}_{3}(3 \times 50 \mathrm{~mL})$ and brine $(3 \times 50 \mathrm{~mL})$, dried over $\mathrm{MgSO}_{4}$, filtrated, and concentrated under vacuum. The crude product was purified by silica gel chromatography with ethyl acetate/petroleum ether (0.5/9.5) to give corresponding compounds $\mathbf{1}-\mathbf{8}$.

Diethyl 4,4-diethyl-3,4-dihydronaphthalene-2,2(1H)-dicarboxylate (1). Colorless oil; yields: 49\% $(\operatorname{method} A), 11 \%(\operatorname{method} B) ;{ }^{1} \mathrm{H}-\mathrm{NMR}\left(\mathrm{CDCl}_{3}\right) \delta_{\mathrm{H}} 0.77\left(\mathrm{t}, J=7.3,6 \mathrm{H}, 2 \mathrm{CH}_{3}\right), 1.22(\mathrm{t}, J=7.2,6 \mathrm{H}$, $\left.2 \mathrm{CH}_{3}\right), 1.52-1.68\left(\mathrm{~m}, 4 \mathrm{H}, 2 \mathrm{CH}_{2}\right), 2.32\left(\mathrm{~s}, 2 \mathrm{H}, \mathrm{CH}_{2}\right), 3.17\left(\mathrm{~s}, 2 \mathrm{H}, \mathrm{CH}_{2}\right), 4.08-4.21\left(\mathrm{~m}, 4 \mathrm{H}, 2 \mathrm{CH}_{2}\right)$,

7.10-7.18 (m, 4H, 4CH). ${ }^{13} \mathrm{C}-\mathrm{NMR}\left(\mathrm{CDCl}_{3}\right) \delta_{\mathrm{C}} 8.3\left(2 \mathrm{CH}_{3}\right), 13.8\left(2 \mathrm{CH}_{3}\right), 33.1\left(\mathrm{CH}_{2}\right), 33.3\left(2 \mathrm{CH}_{2}\right)$, $35.4\left(\mathrm{CH}_{2}\right), 40.2(\mathrm{C}), 52.5(\mathrm{C}), 61.2\left(2 \mathrm{CH}_{2}\right), 125.5(\mathrm{CH}), 126.2(\mathrm{CH}), 126.5(\mathrm{CH}), 128.6(\mathrm{CH}), 134.2$ (C), 141.5 (C), 172.9 (2C). HMRS (ESI): $m / z$ calcd for $\mathrm{C}_{20} \mathrm{H}_{28} \mathrm{O}_{4}\left[\mathrm{M}+\mathrm{H}^{+}\right]$: 333.2060. Found: 333.2061.

Diethyl 2-benzyl-2-(2-ethylbut-2-enyl)malonate (2a/2b) (50:50 inseparable mixture of $Z / E$ isomers). Colorless oil; yields: $10 \%(\operatorname{method} A), 36 \%(\operatorname{method} B) ;{ }^{1} \mathrm{H}-\mathrm{NMR}\left(\mathrm{CDCl}_{3}\right) \delta_{\mathrm{H}} 0.89-0.99\left(\mathrm{~m}, 3 \mathrm{H}, \mathrm{CH}_{3}\right)$, 1.12-1.22 (m, 6H, 2CH $), 1.54-1.64\left(\mathrm{~m}, 3 \mathrm{H}, \mathrm{CH}_{3}\right), 1.93-2.04\left(\mathrm{~m}, 2 \mathrm{H}, \mathrm{CH}_{2}\right), 2.63$ and $2.80(\mathrm{~s}, 2 \mathrm{H}$, $\left.\mathrm{CH}_{2}\right), 3.24$ and $3.26\left(\mathrm{~s}, 2 \mathrm{H}, \mathrm{CH}_{2}\right), 4.03-4.15\left(\mathrm{~m}, 4 \mathrm{H}, 2 \mathrm{CH}_{2}\right), 5.26-5.42(\mathrm{~m}, 1 \mathrm{H}, \mathrm{CH}), 7.11-7.36(\mathrm{~m}$, $5 \mathrm{H}, 5 \mathrm{CH}) .{ }^{13} \mathrm{C}-\mathrm{NMR}\left(\mathrm{CDCl}_{3}\right) \delta_{\mathrm{C}} 12.7\left(\mathrm{CH}_{3}\right), 12.8$ and $13.2\left(\mathrm{CH}_{3}\right), 13.8$ and $13.9\left(2 \mathrm{CH}_{3}\right), 23.3$ and $29.6\left(\mathrm{CH}_{2}\right), 33.5$ and $40.6\left(\mathrm{CH}_{2}\right), 39.1$ and $39.2\left(\mathrm{CH}_{2}\right), 58.9$ and $59.0(\mathrm{C}), 61.1\left(2 \mathrm{CH}_{2}\right), 122.2$ and 
$123.0(\mathrm{CH}), 126.7(\mathrm{CH}), 128.0(2 \mathrm{CH}), 130.1(2 \mathrm{CH}), 130.2(\mathrm{C}), 136.8$ and $137.3(\mathrm{C}), 171.5$ and 171.6 (2C). HMRS (ESI): $m / z$ calcd for $\mathrm{C}_{20} \mathrm{H}_{28} \mathrm{O}_{4}\left[\mathrm{M}+\mathrm{H}^{+}\right]$: 333.2060 . Found: 333.2063 .

Diethyl 2'H-spiro[cyclohexane-1,1'-naphtalene]-3',3'(4'H)-dicarboxylate (3). [34] Colorless oil; yields: 52\% $(\operatorname{method} A), 17 \%(\operatorname{method} B) ;{ }^{1} \mathrm{H}-\mathrm{NMR}\left(\mathrm{CDCl}_{3}\right) \delta_{\mathrm{H}} 1.22\left(\mathrm{t}, J=7.1,6 \mathrm{H}, 2 \mathrm{CH}_{3}\right), 1.47-1.80$ $\left(\mathrm{m}, 10 \mathrm{H}, 5 \mathrm{CH}_{2}\right), 2.46\left(\mathrm{~s}, 2 \mathrm{H}, \mathrm{CH}_{2}\right), 3.19\left(\mathrm{~s}, 2 \mathrm{H}, \mathrm{CH}_{2}\right), 4.14\left(\mathrm{q}, J=7.1,2 \mathrm{H}, \mathrm{CH}_{2}\right), 4.15$ (q, $J=7.1,2 \mathrm{H}$, $\left.\mathrm{CH}_{2}\right), 7.10-7.23(\mathrm{~m}, 3 \mathrm{H}, 3 \mathrm{CH}), 7.35-7.39(\mathrm{~m}, 1 \mathrm{H}, 1 \mathrm{CH}) .{ }^{13} \mathrm{C}-\mathrm{NMR}\left(\mathrm{CDCl}_{3}\right) \delta_{\mathrm{C}} 13.9\left(2 \mathrm{CH}_{3}\right), 21.9$ $\left(2 \mathrm{CH}_{2}\right), 25.9\left(\mathrm{CH}_{2}\right), 34.9\left(\mathrm{CH}_{2}\right), 35.6\left(\mathrm{CH}_{2}\right), 36.8(\mathrm{C}), 39.6\left(2 \mathrm{CH}_{2}\right), 52.4(\mathrm{C}), 61.26\left(2 \mathrm{CH}_{2}\right), 125.8$ $(\mathrm{CH}), 126.1(\mathrm{CH}), 126.5(\mathrm{CH}), 128.7(\mathrm{CH}), 133.4(\mathrm{C}), 144.0(\mathrm{C}), 171.8(2 \mathrm{C})$. Anal. Calcd for $\mathrm{C}_{21} \mathrm{H}_{28} \mathrm{O}_{4}$ : C, 73.23; H, 8.19. Found: C, 73.40; H, 8.50.

Diethyl 2-benzyl-2-(cyclohexenylmethyl)malonate (4). [34] Colorless oil; yields: 11\% (method A), 31\% $(\operatorname{method} B) ;{ }^{1} \mathrm{H}-\mathrm{NMR}\left(\mathrm{CDCl}_{3}\right) \delta_{\mathrm{H}} 1.20\left(\mathrm{t}, J=7.1,6 \mathrm{H}, 2 \mathrm{CH}_{3}\right), 1.55-1.59\left(\mathrm{~m}, 4 \mathrm{H}, 2 \mathrm{CH}_{2}\right), 1.90-2.00(\mathrm{~m}$, $\left.4 \mathrm{H}, 2 \mathrm{CH}_{2}\right), 2.58\left(\mathrm{~s}, 2 \mathrm{H}, \mathrm{CH}_{2}\right), 3.26\left(\mathrm{~s}, 2 \mathrm{H}, \mathrm{CH}_{2}\right), 4.12\left(\mathrm{q}, J=7.1,4 \mathrm{H}, 2 \mathrm{CH}_{2}\right), 5.52(\mathrm{~s}, 1 \mathrm{H}, 1 \mathrm{CH})$, 7.11-7.24 (m, 5H, 5CH). ${ }^{13} \mathrm{C}-\mathrm{NMR}\left(\mathrm{CDCl}_{3}\right) \delta_{\mathrm{C}} 13.9\left(2 \mathrm{CH}_{3}\right), 22.1\left(\mathrm{CH}_{2}\right), 23.0\left(\mathrm{CH}_{2}\right), 25.5\left(\mathrm{CH}_{2}\right), 29.2$ $\left(\mathrm{CH}_{2}\right), 39.0\left(\mathrm{CH}_{2}\right), 41.4\left(\mathrm{CH}_{2}\right), 58.7(\mathrm{C}), 61.0\left(2 \mathrm{CH}_{2}\right), 126.4(\mathrm{CH}), 126.7(\mathrm{CH}), 128.0(2 \mathrm{CH}), 130.1$ (2CH), 133.1 (C), 136.7 (C), 171.4 (2C). Anal. Calcd for $\mathrm{C}_{21} \mathrm{H}_{28} \mathrm{O}_{4}$ : C, 73.23; H, 8.19. Found: C, $72.95 ; \mathrm{H}, 8.35$.

Diethyl 2-(2-ethylbut-2-enyl)-2-methylmalonate (5a/5b) (50:50 inseparable mixture of $Z / E$ isomers). Colorless oil; yields: 47\% (method B); ${ }^{1} \mathrm{H}-\mathrm{NMR}\left(\mathrm{CDCl}_{3}\right) \delta_{\mathrm{H}} 0.81-0.93\left(\mathrm{~m}, 3 \mathrm{H}, \mathrm{CH}_{3}\right), 1.14-1.21(\mathrm{~m}$, $\left.6 \mathrm{H}, 2 \mathrm{CH}_{3}\right), 1.27\left(\mathrm{~s}, 3 \mathrm{H}, \mathrm{CH}_{3}\right), 1.48-1.53\left(\mathrm{~m}, 3 \mathrm{H}, \mathrm{CH}_{3}\right), 1.65-1.96\left(\mathrm{~m}, 2 \mathrm{H}, \mathrm{CH}_{2}\right), 2.57$ and $2.71(\mathrm{~s}, 2 \mathrm{H}$, $\left.\mathrm{CH}_{2}\right), 4.04-4.15\left(\mathrm{~m} ; 4 \mathrm{H}, 2 \mathrm{CH}_{2}\right), 5.13$ and $5.34(\mathrm{~m}, 1 \mathrm{H}, 1 \mathrm{CH}) .{ }^{13} \mathrm{C}-\mathrm{NMR}\left(\mathrm{CDCl}_{3}\right) \delta_{\mathrm{C}} 12.4\left(\mathrm{CH}_{3}\right), 12.6$ and $12.9\left(\mathrm{CH}_{3}\right), 13.7$ and $13.8\left(\mathrm{CH}_{3}\right), 19.2$ and $19.7\left(\mathrm{CH}_{3}\right), 22.9$ and $29.7\left(\mathrm{CH}_{2}\right), 33.6$ and $40.8\left(\mathrm{CH}_{2}\right)$, 53.2 and $53.4(\mathrm{C}), 60.9$ and $61.0\left(2 \mathrm{CH}_{2}\right), 122.4$ and $123.4(\mathrm{CH}), 136.6$ and $136.8(\mathrm{C}), 172.3$ and 172.5 (2C). HMRS (ESI): $m / z$ calcd for $\mathrm{C}_{14} \mathrm{H}_{24} \mathrm{O}_{4}\left[\mathrm{M}+\mathrm{H}^{+}\right]: 257.1747$. Found: 257.1743.

Diethyl 2-(cyclohexenylmethyl)-2-methylmalonate (6). Colorless oil; yields: 46\% (method B); ${ }^{1} \mathrm{H}-\mathrm{NMR}$ $\left(\mathrm{CDCl}_{3}\right) \delta_{\mathrm{H}} 1.23\left(\mathrm{t}, J=7.1 \mathrm{~Hz}, 6 \mathrm{H}, 2 \mathrm{CH}_{3}\right), 1.34\left(\mathrm{~s}, 3 \mathrm{H}, \mathrm{CH}_{3}\right), 1.44-1.58\left(\mathrm{~m}, 4 \mathrm{H}, 2 \mathrm{CH}_{2}\right), 1.73-2.03(\mathrm{~m}$, $\left.4 \mathrm{H}, 2 \mathrm{CH}_{2}\right), 2.58\left(\mathrm{~s}, 2 \mathrm{H}, \mathrm{CH}_{2}\right), 4.15\left(\mathrm{q}, J=7.1,2 \mathrm{CH}_{2}\right), 5.43(\mathrm{~s}, 1 \mathrm{H}, 1 \mathrm{CH}) .{ }^{13} \mathrm{C}-\mathrm{NMR}\left(\mathrm{CDCl}_{3}\right) \delta_{\mathrm{C}} 14.0$ $\left(2 \mathrm{CH}_{3}\right), 19.9\left(\mathrm{CH}_{3}\right), 22.0\left(\mathrm{CH}_{2}\right), 22.9\left(\mathrm{CH}_{2}\right), 25.4\left(\mathrm{CH}_{2}\right), 29.2\left(\mathrm{CH}_{2}\right), 43.7\left(\mathrm{CH}_{2}\right), 53.3(\mathrm{C}), 61.1$ $\left(2 \mathrm{CH}_{2}\right), 126.6(\mathrm{CH}), 132.9(\mathrm{C}), 172.6(2 \mathrm{C})$. HMRS (ESI): $\mathrm{m} / z$ calcd for $\mathrm{C}_{15} \mathrm{H}_{24} \mathrm{O}_{4}\left[\mathrm{M}+\mathrm{H}^{+}\right]: 269.1747$. Found: 269.1754 .

Diethyl 3,3-diethyl-4-methylenecyclopentane-1,1-dicarboxylate (7). Colorless oil; yields: 26\% (method B); ${ }^{1} \mathrm{H}-\mathrm{NMR}\left(\mathrm{CDCl}_{3}\right) \delta_{\mathrm{H}} 0.79\left(\mathrm{t}, J=7.3,6 \mathrm{H}, 2 \mathrm{CH}_{3}\right), 1.24\left(\mathrm{t}, J=7.1,6 \mathrm{H}, 2 \mathrm{CH}_{3}\right), 1.33-1.41(\mathrm{~m}, 4 \mathrm{H}$, $\left.2 \mathrm{CH}_{2}\right), 2.29\left(\mathrm{~s}, 2 \mathrm{H}, \mathrm{CH}_{2}\right), 2.98-3.00\left(\mathrm{~m}, 2 \mathrm{H}, \mathrm{CH}_{2}\right), 4.17$ (q, $\left.J=7.1,4 \mathrm{H}, 2 \mathrm{CH}_{2}\right), 4.65(\mathrm{bs}, 1 \mathrm{H}, \mathrm{CH})$, $4.95(\mathrm{bs}, 1 \mathrm{H}, \mathrm{CH}) .{ }^{13} \mathrm{C}-\mathrm{NMR}\left(\mathrm{CDCl}_{3}\right) \delta_{\mathrm{C}} 8.6\left(2 \mathrm{CH}_{3}\right), 14.0\left(2 \mathrm{CH}_{3}\right), 29.9\left(2 \mathrm{CH}_{2}\right), 41.8\left(\mathrm{CH}_{2}\right), 43.3$ $\left(\mathrm{CH}_{2}\right), 48.5(\mathrm{C}), 57.3(\mathrm{C}), 61.4\left(2 \mathrm{CH}_{2}\right), 106.0\left(\mathrm{CH}_{2}\right), 154.8(\mathrm{C}), 172.3(2 \mathrm{C})$. HMRS (ESI): $\mathrm{m} / z$ calcd for $\mathrm{C}_{16} \mathrm{H}_{26} \mathrm{O}_{4}\left[\mathrm{M}+\mathrm{H}^{+}\right]$: 283.1904. Found: 283.1906.

Diethyl 4-methylenespiro[4.5] decane-2,2-dicarboxylate (8). Colorless oil; yields: 68\% (method B); ${ }^{1} \mathrm{H}-\mathrm{NMR}\left(\mathrm{CDCl}_{3}\right) \delta_{\mathrm{H}} 1.22\left(\mathrm{t}, J=7.2,6 \mathrm{H}, 2 \mathrm{CH}_{3}\right), 1.33-1.66\left(\mathrm{~m}, 10 \mathrm{H}, 5 \mathrm{CH}_{2}\right), 2.33\left(\mathrm{~s}, 2 \mathrm{H}, \mathrm{CH}_{2}\right), 3.01$ 
(bs, 2H, $\mathrm{CH}_{2}$ ), 4.15 (q, $\left.J=7.1,4 \mathrm{H}, 2 \mathrm{CH}_{2}\right), 4.77$ (bs, $\left.1 \mathrm{H}, \mathrm{CH}\right), 4.87$ (bs, $\left.1 \mathrm{H}, \mathrm{CH}\right) .{ }^{13} \mathrm{C}-\mathrm{NMR}\left(\mathrm{CDCl}_{3}\right)$ $\delta_{\mathrm{C}} 13.9\left(2 \mathrm{CH}_{3}\right), 23.2\left(2 \mathrm{CH}_{2}\right), 25.8\left(\mathrm{CH}_{2}\right), 38.0\left(2 \mathrm{CH}_{2}\right), 40.8\left(\mathrm{CH}_{2}\right), 42.6\left(\mathrm{CH}_{2}\right), 45.6(\mathrm{C}), 57.9(\mathrm{C})$, $61.4\left(2 \mathrm{CH}_{2}\right), 104.6\left(\mathrm{CH}_{2}\right), 158.4(\mathrm{C}), 172.1$ (2C). HMRS (ESI): $m / z$ calcd for $\mathrm{C}_{17} \mathrm{H}_{26} \mathrm{O}_{4}\left[\mathrm{M}+\mathrm{H}^{+}\right]$: 295.1904. Found: 295.1903.

\subsection{General Procedure for the Synthesis of Thiobarbituric Acids 9-16}

Thiourea (1.25 g, $16.38 \mathrm{mmol}, 6$ equiv.) was added to a solution of malonate $\mathbf{1 - 8}(2.73 \mathrm{mmol}$, 1 equiv.) in dry DMSO $(3 \mathrm{~mL})$. Then, a solution $1 \mathrm{M}$ of potassium tert-butoxide $(0.67 \mathrm{~g}, 6.0 \mathrm{mmol}$, 2.2 equiv.) was added dropwise. The solution was stirred for $4 \mathrm{~h}$ under inert atmosphere and at $\mathrm{rt}$ (starting from malonates $1,3,7,8$ ) or at $50{ }^{\circ} \mathrm{C}$ (starting from malonates $2, \mathbf{4}, \mathbf{5}, \mathbf{6}$ ). The solution was diluted with ethyl acetate $(15 \mathrm{~mL})$ and washed with a solution of $1 \mathrm{~N}$ hydrochloric acid. The layers were separated and the aqueous phase was extracted with ethyl acetate. The collected organic phase was washed with brine, dried over anhydrous $\mathrm{Na}_{2} \mathrm{SO}_{4}$, filtered and the solvent was removed in vacuo. The residue was purified with column chromatography $\left(\mathrm{CH}_{2} \mathrm{Cl}_{2} /\right.$ petroleum ether, 8:2), affording the corresponding thiobarbituric acids 9-16.

4,4-Diethyl-2'-thioxo-3,4-dihydro-1H,2'H-spiro[naphthalene-2,5'-pyrimidine]-4',6'(1'H,3'H)-dione (9). White solid; m.p. $151{ }^{\circ} \mathrm{C}$ (cyclohexane); yields: 53\% ${ }^{1} \mathrm{H}-\mathrm{NMR}\left(\mathrm{CDCl}_{3}\right) \delta_{\mathrm{H}} 0.76\left(\mathrm{t}, J=7.4,6 \mathrm{H}, 2 \mathrm{CH}_{3}\right)$, 1.67-1.80 (m, 4H, 2CH$), 2.23\left(\mathrm{~s}, 2 \mathrm{H}, \mathrm{CH}_{2}\right), 3.28\left(\mathrm{~s}, 2 \mathrm{H}, \mathrm{CH}_{2}\right), 7.12-7.36(\mathrm{~m}, 4 \mathrm{H}, 4 \mathrm{CH}), 8.99$ (bs, $2 \mathrm{H}) .{ }^{13} \mathrm{C}-\mathrm{NMR}\left(\mathrm{CDCl}_{3}\right) \delta_{\mathrm{C}} 8.4\left(2 \mathrm{CH}_{3}\right), 31.5\left(2 \mathrm{CH}_{2}\right), 34.3\left(\mathrm{CH}_{2}\right), 38.2\left(\mathrm{CH}_{2}\right), 52.2(\mathrm{C}), 53.4(\mathrm{C}), 126.0$ $(\mathrm{CH}), 126.2(\mathrm{CH}), 126.8(\mathrm{CH}), 128.5(\mathrm{CH}), 132.4(\mathrm{C}), 140.9(\mathrm{C}), 170.4(2 \mathrm{C}), 176.0(\mathrm{C})$. HMRS (ESI): $m / z$ calcd for $\mathrm{C}_{17} \mathrm{H}_{20} \mathrm{~N}_{2} \mathrm{O}_{2} \mathrm{~S}\left[\mathrm{M}+\mathrm{H}^{+}\right]: 317.1318$. Found: 317.1317.

5-Benzyl-5-(2-ethylbut-2-enyl)-2-thioxo-dihydropyrimidine-4,6(1H,5H)-dione (10a/10b) (50:50 inseparable mixture of $Z / E$ isomers). White solid; m.p. $182{ }^{\circ} \mathrm{C}$ (cyclohexane); yields: $46 \%{ }^{1} \mathrm{H}-\mathrm{NMR}\left(\mathrm{CDCl}_{3}\right) \delta_{\mathrm{H}}$ 0.90-0.99 (m, 3H, $\left.\mathrm{CH}_{3}\right), 1.53-1.66\left(\mathrm{~m}, 3 \mathrm{H}, \mathrm{CH}_{3}\right), 1.85-2.02\left(\mathrm{~m}, 2 \mathrm{H}, \mathrm{CH}_{2}\right), 2.87$ and $3.00(\mathrm{~s}, 2 \mathrm{H}$, $\left.\mathrm{CH}_{2}\right), 3.30$ and $3.38\left(\mathrm{~s}, 2 \mathrm{H}, \mathrm{CH}_{2}\right), 5.19-5.30$ and 5.41-5.52 (m, $\left.1 \mathrm{H}, \mathrm{CH}\right), 7.07-7.24(\mathrm{~m}, 5 \mathrm{H}, 5 \mathrm{CH})$, $8.84(\mathrm{bs}, 2 \mathrm{H}) .{ }^{13} \mathrm{C}-\mathrm{NMR}\left(\mathrm{CDCl}_{3}\right) \delta_{\mathrm{C}} 12.6$ and $13.0\left(\mathrm{CH}_{3}\right), 13.4$ and $13.7\left(\mathrm{CH}_{3}\right), 23.4$ and $29.9\left(\mathrm{CH}_{2}\right)$, 39.1 and $44.9\left(\mathrm{CH}_{2}\right), 45.0$ and $45.2\left(\mathrm{CH}_{2}\right), 58.0$ and $59.0(\mathrm{C}), 124.6$ and $124.8(\mathrm{CH}), 127.9(\mathrm{CH}), 128.9$ $(2 \mathrm{CH}), 129.5$ and $129.6(2 \mathrm{CH}), 134.2$ and $134.3(\mathrm{C}), 134.7$ and $135.7(\mathrm{C}), 169.6(2 \mathrm{C}), 175.3(\mathrm{C}) . \mathrm{m} / \mathrm{z}$ calcd for $\mathrm{C}_{17} \mathrm{H}_{20} \mathrm{~N}_{2} \mathrm{O}_{2} \mathrm{~S}\left[\mathrm{M}+\mathrm{H}^{+}\right]$: 317.1318. Found: 317.1323 .

2"-Thioxo-2"H,4'H-dispiro[cyclohexane-1,1'-naphtalene-3',5"-pyrimidine]-4",6"(1"H,3"H)-dione (11). White solid; m.p. $200-202{ }^{\circ} \mathrm{C}$ (ethyl alcohol); yields: 64\% ${ }^{1} \mathrm{H}-\mathrm{NMR}\left(\mathrm{CDCl}_{3}\right) \delta_{\mathrm{H}} 1.49-1.84(\mathrm{~m}, 10 \mathrm{H}$, $\left.5 \mathrm{CH}_{2}\right), 2.35\left(\mathrm{~s}, 2 \mathrm{H}, \mathrm{CH}_{2}\right), 3.31\left(\mathrm{~s}, 2 \mathrm{H}, \mathrm{CH}_{2}\right), 7.12-7.41(\mathrm{~m}, 4 \mathrm{H}, 4 \mathrm{CH}), 9.33(\mathrm{bs}, 2 \mathrm{H}, 2 \mathrm{NH}) .{ }^{13} \mathrm{C}-\mathrm{NMR}$ $\left(\mathrm{CDCl}_{3}\right) \delta_{\mathrm{C}} 22.0\left(2 \mathrm{CH}_{2}\right), 25.7\left(\mathrm{CH}_{2}\right), 33.6\left(\mathrm{CH}_{2}\right), 37.8(\mathrm{C}), 38.1\left(2 \mathrm{CH}_{2}\right), 38.3\left(\mathrm{CH}_{2}\right), 52.2(\mathrm{C}), 125.1$ $(\mathrm{CH}), 126.1(\mathrm{CH}), 127.2(\mathrm{CH}), 128.5(\mathrm{CH}), 132.1(\mathrm{C}), 143.8(\mathrm{C}), 170.2(2 \mathrm{C}), 176.0(\mathrm{C})$. HMRS (ESI): $m / z$ calcd for $\mathrm{C}_{18} \mathrm{H}_{20} \mathrm{~N}_{2} \mathrm{O}_{2} \mathrm{~S}\left[\mathrm{M}+\mathrm{H}^{+}\right]: 329.1318$. Found: 329.1317.

5-Benzyl-5-(cyclohexenylmethyl)-2-thioxo-dihydropyrimidine-4,6(1H,5H)-dione (12). Colorless oil; yields: $88 \%{ }^{1} \mathrm{H}-\mathrm{NMR}\left(\mathrm{CDCl}_{3}\right) \delta_{\mathrm{H}} 1.35-2.04\left(\mathrm{~m}, 8 \mathrm{H}, 4 \mathrm{CH}_{2}\right), 2.82\left(\mathrm{~s}, 2 \mathrm{H}, \mathrm{CH}_{2}\right), 3.31\left(\mathrm{~s}, 2 \mathrm{H}, \mathrm{CH}_{2}\right), 5.50$ $(\mathrm{s}, 1 \mathrm{H}, 1 \mathrm{CH}), 7.13-7.26(\mathrm{~m}, 5 \mathrm{H}, 5 \mathrm{CH}), 8.98(\mathrm{bs}, 2 \mathrm{H}, 2 \mathrm{NH}) .{ }^{13} \mathrm{C}-\mathrm{NMR}\left(\mathrm{CDCl}_{3}\right) \delta_{\mathrm{C}} 21.9\left(\mathrm{CH}_{2}\right), 22.8$ 
$\left(\mathrm{CH}_{2}\right), 23.6\left(\mathrm{CH}_{2}\right), 29.8\left(\mathrm{CH}_{2}\right), 44.5\left(\mathrm{CH}_{2}\right), 47.6\left(\mathrm{CH}_{2}\right), 58.9(\mathrm{C}), 127.7(\mathrm{CH}), 127.8(\mathrm{CH}), 128.8$ $(2 \mathrm{CH}), 129.5(2 \mathrm{CH}), 131.5$ (C), 134.3 (C), 169.7 (2C), 175.4 (C). HMRS (ESI): $\mathrm{m} / z$ calcd for $\mathrm{C}_{18} \mathrm{H}_{20} \mathrm{~N}_{2} \mathrm{O}_{2} \mathrm{~S}\left[\mathrm{M}+\mathrm{NH}_{4}^{+}\right]$: 346.1584. Found: 346.1579 .

5-(2-Ethylbut-2-enyl)-5-methyl-2-thioxo-dihydropyrimidine-4,6(1H,5H)-dione (13a/13b) (50:50 inseparable mixture of $Z / E$ isomers). Colorless oil; yields: $75 \%{ }^{1} \mathrm{H}-\mathrm{NMR}\left(\mathrm{CDCl}_{3}\right) \delta_{\mathrm{H}} 0.87-0.97\left(\mathrm{~m}, 3 \mathrm{H}, \mathrm{CH}_{3}\right)$, 1.54-1.61 (m, 3H, $\left.\mathrm{CH}_{3}\right), 1.57\left(\mathrm{~s}, 3 \mathrm{H}, \mathrm{CH}_{3}\right), 1.80-2.01\left(\mathrm{~m}, 2 \mathrm{H}, \mathrm{CH}_{2}\right), 2.70$ and $2.82\left(\mathrm{~s}, 2 \mathrm{H}, \mathrm{CH}_{2}\right), 5.18$ and $5.47(\mathrm{~m}, 1 \mathrm{H}, \mathrm{CH}), 9.05(\mathrm{bs}, 2 \mathrm{H}, 2 \mathrm{NH}) .{ }^{13} \mathrm{C}-\mathrm{NMR}\left(\mathrm{CDCl}_{3}\right) \delta_{\mathrm{C}} 12.6$ and $13.0\left(\mathrm{CH}_{3}\right), 13.3$ and 13.9 $\left(\mathrm{CH}_{3}\right), 23.1$ and $23.3\left(\mathrm{CH}_{3}\right), 23.5$ and $29.9\left(\mathrm{CH}_{2}\right), 40.4$ and $46.2\left(\mathrm{CH}_{2}\right), 51.0$ and $51.9(\mathrm{C}), 124.5$ and $125.0(\mathrm{CH}), 134.8$ and $135.9(\mathrm{C}), 170.5$ and $170.6(2 \mathrm{C}), 176.0(\mathrm{C})$. Anal. Calcd for $\mathrm{C}_{11} \mathrm{H}_{16} \mathrm{~N}_{2} \mathrm{O}_{2} \mathrm{~S}: \mathrm{C}$, 54.98; H, 6.71; N, 11.66. Found: C, 55.15; H, 6.86; N, 11.63.

5-(Cyclohexenylmethyl)-5-methyl-2-thioxo-dihydropyrimidine-4,6(1H,5H)-dione (14). White solid; m.p. $160-164{ }^{\circ} \mathrm{C}$ (ethyl alcohol); yields: $90 \%{ }^{1} \mathrm{H}-\mathrm{NMR}\left(\mathrm{CDCl}_{3}\right) \delta_{\mathrm{H}} 1.37-1.52\left(\mathrm{~m}, 4 \mathrm{H}, 2 \mathrm{CH}_{2}\right), 1.57$ (s, 3H, $\left.\mathrm{CH}_{3}\right), 1.76-1.98\left(\mathrm{~m}, 4 \mathrm{H}, 2 \mathrm{CH}_{2}\right), 2.65$ (s, 2H, $\left.\mathrm{CH}_{2}\right), 5.44(\mathrm{~s}, 1 \mathrm{H}, 1 \mathrm{CH}), 9.61$ (bs, 2H, 2NH). ${ }^{13} \mathrm{C}-\mathrm{NMR}\left(\mathrm{CDCl}_{3}\right) \delta_{\mathrm{C}} 21.8\left(\mathrm{CH}_{2}\right), 22.8\left(\mathrm{CH}_{2}\right), 23.0\left(\mathrm{CH}_{3}\right), 25.4\left(\mathrm{CH}_{2}\right), 29.7\left(\mathrm{CH}_{2}\right), 48.5\left(\mathrm{CH}_{2}\right), 51.8$ (C), $127.5(\mathrm{CH}), 131.6(\mathrm{C}), 170.9$ (2C), 176.2 (C). HMRS (ESI): $m / z$ calcd for $\mathrm{C}_{12} \mathrm{H}_{16} \mathrm{~N}_{2} \mathrm{O}_{2} \mathrm{~S}\left[\mathrm{M}+\mathrm{H}^{+}\right]$: 253.1005. Found: 253.1007.

2,2-Diethyl-3-methylene-8-thioxo-7,9-diazaspiro[4.5]decane-6,10-dione (15). White solid; m.p. 194-196 ${ }^{\circ} \mathrm{C}$ (cyclohexane); yields: $70 \%{ }^{1} \mathrm{H}-\mathrm{NMR}\left(\mathrm{CDCl}_{3}\right) \delta_{\mathrm{H}} 0.83\left(\mathrm{t}, J=7.4,6 \mathrm{H}, 2 \mathrm{CH}_{3}\right), 1.43-1.70$


$2 \mathrm{H}, 2 \mathrm{NH}) .{ }^{13} \mathrm{C}-\mathrm{NMR}\left(\mathrm{CDCl}_{3}\right) \delta_{\mathrm{C}} 8.7\left(2 \mathrm{CH}_{3}\right), 29.0\left(2 \mathrm{CH}_{2}\right), 44.4\left(\mathrm{CH}_{2}\right), 47.2\left(\mathrm{CH}_{2}\right), 49.9(\mathrm{C}), 54.3(\mathrm{C})$, $107.4\left(\mathrm{CH}_{2}\right), 153.4(\mathrm{C}), 170.7(2 \mathrm{C}), 176.1(\mathrm{C})$. HMRS (ESI): $m / z$ calcd for $\mathrm{C}_{13} \mathrm{H}_{18} \mathrm{~N}_{2} \mathrm{O}_{2} \mathrm{~S}\left[\mathrm{M}+\mathrm{NH}_{4}^{+}\right]$: 284.1427. Found: 284.1434.

14-Methylene-3-thioxo-2,4-diazadispiro[5.1.5.2]pentadecane-1,5-dione (16). White solid; m.p. $177{ }^{\circ} \mathrm{C}$ (isopropanol); yields: $54 \%{ }^{1} \mathrm{H}-\mathrm{NMR}\left(\mathrm{CDCl}_{3}\right) \delta_{\mathrm{H}} 1.22-1.47\left(\mathrm{~m}, 6 \mathrm{H}, 2 \mathrm{CH}_{3}\right), 1.66-1.77\left(\mathrm{~m}, 4 \mathrm{H}, 2 \mathrm{CH}_{2}\right)$, $2.33\left(\mathrm{~s}, 2 \mathrm{H}, \mathrm{CH}_{2}\right), 3.06$ (s, 2H, $\left.\mathrm{CH}_{2}\right), 4.89-4.93$ (m, 2H, $\left.\mathrm{CH}_{2}\right), 9.09$ (bs, 2H, 2NH). ${ }^{13} \mathrm{C}-\mathrm{NMR}\left(\mathrm{CDCl}_{3}\right)$ $\delta_{\mathrm{C}} 23.2\left(2 \mathrm{CH}_{2}\right), 25.7\left(\mathrm{CH}_{2}\right), 37.5\left(2 \mathrm{CH}_{2}\right), 44.0\left(\mathrm{CH}_{2}\right), 45.2\left(\mathrm{CH}_{2}\right), 46.8(\mathrm{C}), 55.0(\mathrm{C}), 105.4\left(\mathrm{CH}_{2}\right)$, 157.4 (C), 170.7 (2C), 176.2 (C). HMRS (ESI): $m / z$ calcd for $\mathrm{C}_{14} \mathrm{H}_{18} \mathrm{~N}_{2} \mathrm{O}_{2} \mathrm{~S}\left[\mathrm{M}+\mathrm{NH}_{4}^{+}\right]$: 296.1427. Found: 296.1422.

\subsection{General Procedure for Salification of Barbituric Acids to Barbiturate Potassium Salts 17-24}

A suspension of potassium hydroxide $(0.02 \mathrm{~g}, 0.36 \mathrm{mmol}, 1$ equiv. $)$ in isopropanol $(5 \mathrm{~mL})$ was stirred under inert atmosphere. The corresponding barbituric acid 9-16 (0.36 mmol, 1 equiv.) was added, and reaction was monitored by TLC until the barbituric acid disappeared. Isopropanol was removed in vacuo, and corresponding barbiturates 17-24 were obtained without further purification.

Potassium 4,4-diethyl-4',6'-dioxo-1',3,4,6'-tetrahydro-1H,4'H-spiro[naphthalene-2,5'-pyrimidine]-2'thiolate (17). White solid; m.p. 161-163 ${ }^{\circ} \mathrm{C}$ (isopropanol); yields: 77\%; ${ }^{1} \mathrm{H}-\mathrm{NMR}\left(\mathrm{D}_{2} \mathrm{O}\right) \delta_{\mathrm{H}} 0.72$ (s, $\left.3 \mathrm{H}, \mathrm{CH}_{3}\right), 0.99\left(\mathrm{~s}, 3 \mathrm{H}, \mathrm{CH}_{3}\right), 1.42-1.79\left(\mathrm{~m}, 4 \mathrm{H}, 2 \mathrm{CH}_{2}\right), 2.38\left(\mathrm{~d}, J=15.4,1 \mathrm{H}, \mathrm{CH}_{2}\right), 2.53(\mathrm{~d}, J=15.4$, $\left.1 \mathrm{H}, \mathrm{CH}_{2}\right), 3.13\left(\mathrm{~d}, J=16.4,1 \mathrm{H}, \mathrm{CH}_{2}\right), 3.40\left(\mathrm{~d}, J=16.4,1 \mathrm{H}, \mathrm{CH}_{2}\right), 7.35-7.41(\mathrm{~m}, 4 \mathrm{H}, 4 \mathrm{CH}) .{ }^{13} \mathrm{C}-\mathrm{NMR}$ 
$\left(\mathrm{D}_{2} \mathrm{O}\right) \delta_{\mathrm{C}} 8.4\left(\mathrm{CH}_{3}\right), 8.5\left(\mathrm{CH}_{3}\right), 32.9\left(\mathrm{CH}_{2}\right), 35.1\left(\mathrm{CH}_{2}\right), 35.2\left(\mathrm{CH}_{2}\right), 35.8\left(\mathrm{CH}_{2}\right), 41.2(\mathrm{C}), 57.2(\mathrm{C})$, $126.5(\mathrm{CH}), 127.0(\mathrm{CH}), 127.9(\mathrm{CH}), 129.1(\mathrm{CH}), 136.2(\mathrm{C}), 142.8(\mathrm{C}), 177.0(2 \mathrm{C}), 178.9(\mathrm{C})$. HMRS (ESI): $m / z$ calcd for $\mathrm{C}_{17} \mathrm{H}_{19} \mathrm{~N}_{2} \mathrm{O}_{2} \mathrm{~S}^{-} \mathrm{M}: 315.1173$. Found: 315.1183 .

Potassium 5-benzyl-5-[2-ethylbut-2-en-1-yl]-4,6-thioxo-1,4,5,6-tetrahydropyrimidine-2-thiolate (18a/ 18b) (50:50 inseparable mixture of $Z / E$ isomers). White solid; m.p. $142-144{ }^{\circ} \mathrm{C}$ (isopropanol); yields: $78 \%$; ${ }^{1} \mathrm{H}-\mathrm{NMR}\left(\mathrm{D}_{2} \mathrm{O}\right) \delta_{\mathrm{H}} 0.98-1.05\left(\mathrm{~m}, 3 \mathrm{H}, \mathrm{CH}_{3}\right), 1.61-1.73\left(\mathrm{~m}, 3 \mathrm{H}, \mathrm{CH}_{2}\right), 1.93-2.12\left(\mathrm{~m}, 2 \mathrm{H}, \mathrm{CH}_{2}\right), 2.89$ and $3.01\left(\mathrm{~s}, 2 \mathrm{H}, \mathrm{CH}_{2}\right), 3.28$ and $3.38\left(\mathrm{~s}, 2 \mathrm{H}, \mathrm{CH}_{2}\right), 5.11$ and $5.51(\mathrm{bs}, 1 \mathrm{H}, 1 \mathrm{CH}), 7.22-7.39(\mathrm{~m}, 5 \mathrm{H}$, 5CH). ${ }^{13} \mathrm{C}-\mathrm{NMR}\left(\mathrm{D}_{2} \mathrm{O}\right) \delta_{\mathrm{C}} 12.6$ and $12.7\left(\mathrm{CH}_{3}\right), 13.2$ and $13.3\left(\mathrm{CH}_{3}\right), 23.6$ and $29.8\left(\mathrm{CH}_{2}\right), 39.3$ and $44.8\left(\mathrm{CH}_{2}\right), 45.0$ and $45.9\left(\mathrm{CH}_{2}\right), 57.6(\mathrm{C}), 122.7$ and $123.8(\mathrm{CH}), 128.0(\mathrm{CH}), 129.1(2 \mathrm{CH}), 129.9$ (2CH), 135.9 (C), 138.0 (C), 172.9 (C), 179.6 (2C). HMRS (ESI): $m / z$ calcd for $\mathrm{C}_{17} \mathrm{H}_{19} \mathrm{~N}_{2} \mathrm{O}_{2} \mathrm{~S}^{-} \mathrm{M}$ : 315.1173. Found: 315.1180 .

Potassium 4",6"-dioxo-1",6"-dihydro-4'H,4"H-dispiro[cyclohexane-1,1'-naphtalene-3',5"-pyrimidine]2"-thiolate (19). White solid; m.p. $216-218{ }^{\circ} \mathrm{C}$ (isopropanol); yields: 70\%; ${ }^{1} \mathrm{H}-\mathrm{NMR}\left(\mathrm{D}_{2} \mathrm{O}\right) \delta_{\mathrm{H}} 1.38-2.25$ (m, 10H, 5CH $), 2.40$ (bs, 1H, $\left.\mathrm{CH}_{2}\right), 3.08-3.68\left(\mathrm{~m}, 3 \mathrm{H}, \mathrm{CH}_{2}\right), 7.40-7.58(\mathrm{~m}, 3 \mathrm{H}, 3 \mathrm{CH}), 7.72-7.78(\mathrm{~m}$, $1 \mathrm{H}, 1 \mathrm{CH}) .{ }^{13} \mathrm{C}-\mathrm{NMR}\left(\mathrm{D}_{2} \mathrm{O}\right) \delta_{\mathrm{C}} 22.1\left(\mathrm{CH}_{2}\right), 22.4\left(\mathrm{CH}_{2}\right), 26.0\left(\mathrm{CH}_{2}\right), 35.8\left(\mathrm{CH}_{2}\right), 37.6\left(\mathrm{CH}_{2}\right), 37.7(\mathrm{C})$, $38.2\left(\mathrm{CH}_{2}\right), 42.0\left(\mathrm{CH}_{2}\right), 56.9(\mathrm{C}), 126.8(\mathrm{CH}), 127.2(\mathrm{CH}), 127.3(\mathrm{CH}), 129.3(\mathrm{CH}), 135.3(\mathrm{C}), 144.7$ (C), 176.5 (C), 178.8 (C), 181.5 (C). HMRS (ESI): $m / z$ calcd for $\mathrm{C}_{18} \mathrm{H}_{19} \mathrm{~N}_{2} \mathrm{O}_{2} \mathrm{~S}^{-} \mathrm{M}: 327.1173$. Found: 327.1184 .

Potassium 5-benzyl-5-(cyclohex-1-en-1-ylmethyl)-4,6-dioxo-1,4,5,6-tetrahydropyrimidine-2-thiolate (20). White solid; m.p. $143{ }^{\circ} \mathrm{C}$ (isopropanol); yields: 84\% ${ }^{1} \mathrm{H}-\mathrm{NMR}\left(\mathrm{D}_{2} \mathrm{O}\right) \delta_{\mathrm{H}} 1.36-1.60\left(\mathrm{~m}, 4 \mathrm{H}, 2 \mathrm{CH}_{2}\right)$, 1.74-2.00 (m, 4H, 2CH $), 2.70\left(\mathrm{~s}, 2 \mathrm{H}, \mathrm{CH}_{2}\right), 3.18\left(\mathrm{~s}, 2 \mathrm{H}, \mathrm{CH}_{2}\right), 5.39(\mathrm{~s}, 1 \mathrm{H}, 1 \mathrm{CH}), 7.06-7.11(\mathrm{~m}, 2 \mathrm{H}$, 2CH), 7.26-7.30 (m, 3H, 3CH). ${ }^{13} \mathrm{C}-\mathrm{NMR}\left(\mathrm{D}_{2} \mathrm{O}\right) \delta_{\mathrm{C}} 22.3\left(\mathrm{CH}_{2}\right), 23.3\left(\mathrm{CH}_{2}\right), 25.7\left(\mathrm{CH}_{2}\right), 29.8\left(\mathrm{CH}_{2}\right)$, $45.5\left(\mathrm{CH}_{2}\right), 47.6\left(\mathrm{CH}_{2}\right), 57.3(\mathrm{C}), 126.2(\mathrm{CH}), 127.8(\mathrm{CH}), 129.1(2 \mathrm{CH}), 129.9(2 \mathrm{CH}), 134.0(\mathrm{C})$, 136.6 (C), 181.5 (2C), 192.6 (C). HMRS (ESI): $m / z$ calcd for $\mathrm{C}_{18} \mathrm{H}_{19} \mathrm{~N}_{2} \mathrm{O}_{2} \mathrm{~S}^{-} \mathrm{M}: 327.1173$. Found: 327.1173 .

Potassium 5-[2-ethylbut-2-en-1-yl]-5-methyl-4,6-dioxo-1,4,5,6-tetrahydropyrimidine-2-thiolate (21a/ 21b) (50:50 inseparable mixture of $Z / E$ isomers). White solid; m.p. $174-176{ }^{\circ} \mathrm{C}$ (isopropanol); yields: $28 \%$ ${ }^{1} \mathrm{H}-\mathrm{NMR}\left(\mathrm{D}_{2} \mathrm{O}\right) \delta_{\mathrm{H}} 0.82-0.98\left(\mathrm{~m}, 3 \mathrm{H}, \mathrm{CH}_{3}\right), 1.32-1.42\left(\mathrm{~m}, 3 \mathrm{H}, \mathrm{CH}_{3}\right), 1.49-1.56\left(\mathrm{~m}, 3 \mathrm{H}, \mathrm{CH}_{3}\right)$, 1.76-2.05 (m, 2H, CH$), 2.54-2.69\left(\mathrm{~m}, 2 \mathrm{H}, \mathrm{CH}_{2}\right), 5.01$ and $5.45(\mathrm{bs}, 1 \mathrm{H}, 1 \mathrm{CH}) .{ }^{13} \mathrm{C}-\mathrm{NMR}\left(\mathrm{D}_{2} \mathrm{O}\right) \delta_{\mathrm{C}}$ 12.8 and $13.0\left(\mathrm{CH}_{3}\right), 13.2$ and $14.0\left(\mathrm{CH}_{3}\right), 21.0$ and $22.7\left(\mathrm{CH}_{3}\right), 23.7$ and $30.2\left(\mathrm{CH}_{2}\right), 38.5$ and 44.8 $\left(\mathrm{CH}_{2}\right), 56.7$ and $57.0(\mathrm{C}), 123.1$ and $123.8(\mathrm{CH}), 138.3$ and $139.1(\mathrm{C}), 177.8$ and $177.9(\mathrm{C}), 180.0$ and 180.1 (C), 181.5 and 181.6 (C). HMRS (ESI): $m / z$ calcd for $\mathrm{C}_{11} \mathrm{H}_{15} \mathrm{~N}_{2} \mathrm{O}_{2} \mathrm{~S}^{-} \mathrm{M}$ : 239.0860. Found: 239.0857 .

Potassium 5-(cyclohex-1-en-1-ylmethyl)-5-methyl-4,6-dioxo-1,4,5,6-tetrahydropyrimidine-2-thiolate (22). White solid; m.p. $177{ }^{\circ} \mathrm{C}$ (isopropanol); yields: 69\% ${ }^{1} \mathrm{H}-\mathrm{NMR}\left(\mathrm{D}_{2} \mathrm{O}\right) \delta_{\mathrm{H}} 1.47\left(\mathrm{~s}, 3 \mathrm{H}, \mathrm{CH}_{3}\right)$, $1.45-1.61\left(\mathrm{~m}, 4 \mathrm{H}, 2 \mathrm{CH}_{2}\right), 1.84-2.09\left(\mathrm{~m}, 4 \mathrm{H}, 2 \mathrm{CH}_{2}\right), 2.55\left(\mathrm{~s}, 2 \mathrm{H}, \mathrm{CH}_{2}\right), 5.41(\mathrm{~s}, 1 \mathrm{H}, 1 \mathrm{CH}) .{ }^{13} \mathrm{C}-\mathrm{NMR}$ $\left(\mathrm{D}_{2} \mathrm{O}\right) \delta_{\mathrm{C}} 22.4\left(\mathrm{CH}_{2}\right), 22.5\left(\mathrm{CH}_{3}\right), 23.3\left(\mathrm{CH}_{2}\right), 25.7\left(\mathrm{CH}_{2}\right), 29.6\left(\mathrm{CH}_{2}\right), 47.4\left(\mathrm{CH}_{2}\right), 56.8(\mathrm{C}), 126.7$ 
(CH), 134.9 (C), 177.9 (2C), 181.6 (C). HMRS (ESI): $m / z$ calcd for $\mathrm{C}_{12} \mathrm{H}_{15} \mathrm{~N}_{2} \mathrm{O}_{2} \mathrm{~S}^{-}$M: 251.0860 . Found: 251.0859.

Potassium 2,2-diethyl-3-methylene-6,10-dioxo-7,9-diazaspiro[4.5]dec-7-ene-8-thiolate (23). White solid; decomp. $270{ }^{\circ} \mathrm{C}$ (isopropanol); yields: 88\% ${ }^{1} \mathrm{H}-\mathrm{NMR}\left(\mathrm{D}_{2} \mathrm{O}\right) \delta_{\mathrm{H}} 0.72-0.83\left(\mathrm{~m}, 6 \mathrm{H}, 2 \mathrm{CH}_{3}\right)$, 1.14-1.53 (m, 4H, 2CH $), 2.27\left(\mathrm{~s}, 2 \mathrm{H}, \mathrm{CH}_{2}\right), 2.84\left(\mathrm{~d}, J=16.3,1 \mathrm{H}, \mathrm{CH}_{2}\right), 3.04\left(\mathrm{~d}, J=16.3,1 \mathrm{H}, \mathrm{CH}_{2}\right)$, $4.72(\mathrm{bs}, 1 \mathrm{H}, \mathrm{CH}), 5.01(\mathrm{bs}, 1 \mathrm{H}, \mathrm{CH}) .{ }^{13} \mathrm{C}-\mathrm{NMR}\left(\mathrm{D}_{2} \mathrm{O}\right) \delta_{\mathrm{C}} 8.6\left(\mathrm{CH}_{3}\right), 8.7\left(\mathrm{CH}_{3}\right), 30.5\left(\mathrm{CH}_{2}\right), 31.0$ $\left(\mathrm{CH}_{2}\right), 41.7\left(\mathrm{CH}_{2}\right), 45.0\left(\mathrm{CH}_{2}\right), 49.1(\mathrm{C}), 62.5(\mathrm{C}), 105.8\left(\mathrm{CH}_{2}\right), 157.2(\mathrm{C}), 176.7(\mathrm{C}), 179.1(\mathrm{C}), 182.1$ (C). HMRS (ESI): $m / z$ calcd for $\mathrm{C}_{13} \mathrm{H}_{17} \mathrm{~N}_{2} \mathrm{O}_{2} \mathrm{~S}^{-} \mathrm{M}: 265.1016$. Found: 265.1025.

Potassium 14-methylene-1,5-dioxo-2,4-diazaspiro[5.1.5.2]pentadec-2-ene-3-thiolate (24). White solid; m.p. $174-176{ }^{\circ} \mathrm{C}$ (isopropanol); yields: 53\% ${ }^{1} \mathrm{H}-\mathrm{NMR}\left(\mathrm{D}_{2} \mathrm{O}\right) \delta_{\mathrm{H}} 1.13-1.65\left(\mathrm{~m}, 10 \mathrm{H}, 5 \mathrm{CH}_{2}\right), 2.25(\mathrm{~d}$, $\left.J=14.0,1 \mathrm{H}, \mathrm{CH}_{2}\right), 2.40\left(\mathrm{~d}, J=14.0,1 \mathrm{H}, \mathrm{CH}_{2}\right), 2.88\left(\mathrm{~d}, J=16.4,1 \mathrm{H}, \mathrm{CH}_{2}\right), 3.04(\mathrm{~d}, J=16.4,1 \mathrm{H}$, $\left.\mathrm{CH}_{2}\right), 4.86$ (bs, $\left.\mathrm{CH}\right), 4.96$ (bs, $\left.\mathrm{CH}\right) .{ }^{13} \mathrm{C}-\mathrm{NMR}\left(\mathrm{D}_{2} \mathrm{O}\right) \delta_{\mathrm{C}} 22.8\left(\mathrm{CH}_{2}\right), 22.9\left(\mathrm{CH}_{2}\right), 37.6\left(\mathrm{CH}_{2}\right), 38.6$ $\left(\mathrm{CH}_{2}\right), 40.1\left(\mathrm{CH}_{2}\right), 44.0\left(\mathrm{CH}_{2}\right), 45.7(\mathrm{C}), 62.3(\mathrm{C}), 104.0\left(\mathrm{CH}_{2}\right), 160.7(\mathrm{C}), 175.9(\mathrm{C}), 178.3(\mathrm{C}) .1 \mathrm{C}$ not observed in these conditions. HMRS (ESI): $m / z$ calcd for $\mathrm{C}_{14} \mathrm{H}_{17} \mathrm{~N}_{2} \mathrm{O}_{2} \mathrm{~S}^{-} \mathrm{M}: 277.1016$. Found: 277.1009.

\section{Conclusions}

We have synthesized eight new functionalized thiobarbiturates by a three steps synthesis, thanks to $\mathrm{Mn}(\mathrm{OAc})_{3}$ radical reactivity. This methodology allows $C$-functionalization of barbituric acid with a wide variety of scaffolds, such as aromatic, aliphatic and spirocyclic moieties. Derivatives thus obtained could be tested for their anesthetic potentialities, but also for targeting anticonvulsive leads.

\section{Acknowledgements}

This work was supported by the Centre National de la Recherche Scientifique and Aix-Marseille University. We would like to express our thanks to V. Remusat for recording the NMR spectra and V. Monnier for recording the mass spectra.

\section{References and Notes}

1. Snider, B.B. Manganese(III)-based oxidative free-radical cyclizations. Chem. Rev. 1996, 96, 339-363.

2. Demir, A.S.; Emrullahoglu, M. Manganese(III) acetate: A versatile reagent in organic chemistry. Curr. Org. Synth. 2007, 4, 321-350.

3. Dombroski, M.A.; Snider, B.B. Manganese(III)-Based oxidative free-radical. Cyclizations of $\gamma, \gamma$-bis(allylic) acetoacetates. Tetrahedron 1992, 48, 1417-1426.

4. Kates, S.A.; Dombroski, M.A.; Snider, B.B. Manganese(III)-based oxidative free-radical cyclization of unsaturated beta-keto esters, 1,3-diketones, and malonate diesters. J. Org. Chem. 1990, 55, 2427-2436.

5. Chuang, C.-P.; Tsai, A.-I. A novel oxidative free radical reaction between 2-amino-1,4benzoquinones and benzoylacetonitriles. Tetrahedron 2007, 63, 9712-9717. 
6. Logoglu, E.; Yilmaz, M.; Katircioglu, H.; Yakut, M.; Mercan, S. Synthesis and biological activity studies of furan derivatives. Med. Chem. Res. 2010, 19, 490-497.

7. Curti, C.; Crozet, M.D.; Vanelle, P. Microwave-assisted manganese(III) acetate based oxidative cyclizations of alkenes with $\beta$-ketosulfones. Tetrahedron 2009, 65, 200-205.

8. Bouhlel, A.; Curti, C.; Dumètre, A.; Laget, M.; Crozet, M.D.; Azas, N.; Vanelle, P. Synthesis and evaluation of original amidoximes as antileishmanial agents. Bioorg. Med. Chem. 2010, 18, 7310-7320.

9. Paloque, L.; Bouhlel, A.; Curti, C.; Dumètre, A.; Verhaeghe, P.; Azas, N.; Vanelle, P. Synthesis and evaluation of monoamidoxime derivatives: Toward new antileishmanial compounds. Eur. J. Med. Chem. 2011, 46, 2984-2991.

10. Biltz, H.; Witteck, H. Alkylated and acylated barbituric acids. Ber. Dtsch. Chem. Ges. 1921, 54, 1035-1059.

11. Clark-Lewis, J.W.; Thompson, M.J. Preparation of 1,3-dimethylbarbituric acid and formation of 5-ethoxycarbonylacetyl-1,3-dimethylbarbituric acid. J. Chem. Soc. 1959, 1628-1629.

12. Nguyen, V.; Nishino, H. Novel synthesis of dihydropyrans and 2,8-dioxabicylo[3.3.0]oct-3-enes using Mn(III)-based oxidative cyclization. Tetrahedron Lett. 2004, 45, 3373-3377.

13. Tsubusaki, T.; Nishino, H. Manganese(III)-mediated facile synthesis of 3,4-dihydro-2(1H)quinolinones: Selectivity of the 6-endo and 5-exo cyclization. Tetrahedron 2009, 65, 9448-9459.

14. Delmas, F.; Gasquet, M.; Timon-David, P.; Madadi, N.; Vanelle, P.; Vaille, A.; Maldonado, J. Synthesis and in vitro anti-protozoan activity of new 5-nitrothiophene oxime ether derivatives. Eur. J. Med. Chem. 1993, 28, 23-27.

15. Baraldi, P.G.; El-Kashef, H.; Farghaly, A.R.; Vanelle, P.; Fruttarolo F. Synthesis of new pyrazolo[4,3-e]-1,2,4-triazolo[1,5-c]pyrimidines and related heterocycles. Tetrahedron 2004, 60, 5093-5104.

16. Boufatah, N.; Gellis, A.; Maldonado, J.; Vanelle, P. Efficient microwave-assisted synthesis of new sulfonylbenzimidazole-4,7-diones: Heterocyclic quinones with potential antitumor activity. Tetrahedron 2004, 60, 9131-9137.

17. Verhaeghe, P.; Azas, N.; Gasquet, M.; Hutter, S.; Ducros, C.; Laget, M.; Rault, S.; Rathelot, P.; Vanelle, P. Synthesis and antiplasmodial activity of new 4-aryl-2-trichloromethylquinazolines. Bioorg. Med. Chem. Lett. 2008, 18, 396-401.

18. Kabri, Y.; Gellis, A.; Vanelle, P. Microwave-assisted synthesis in aqueous medium of new quinazoline derivatives as anticancer agent precursors. Green Chem. 2009, 11, 201-208.

19. Smith, M.C.; Riskin, B.J. The clinical use of barbiturates in neurological disorders. Drugs 1991, $42,365-378$.

20. Nagel, S.; von Heinemann, P.; Heiland, S.; Koziol, J.; Gardner, H.; Wagner, S. Selective MMP-inhibition with Ro 28-2653 in acute experimental stroke-A magnetic resonance imaging efficacy study. Brain Res. 2011, 1368, 264-270.

21. Wang, J.; Medina, C.; Radomski, M.W.; Gilmer, J.F. N-substituted homopiperazine barbiturates as gelatinase inhibitors. Bioorg. Med. Chem. 2011, 19, 4985-4999. 
22. Vijaya, L.S.; Thirupathi, R.Y.; Suresh, K.B.; Narsimha, R.P.; Crooks, P.A.; Rajitha, B. Synthesis and evaluation of chromenyl barbiturates and thiobarbiturates as potential antitubercular agents. Bioorg. Med. Chem. Lett. 2011, 21, 4329-4331.

23. Al-Najjar, B.O.; Wahab, H.A.; Muhammad, T.S.T.; Shu-Chien, A.C.; Noruddin, N.A.A.; Taha, M.O. Discovery of new nanomolar peroxisome proliferator-activated receptor $\gamma$ activators via elaborate ligand-based modeling. Eur. J. Med. Chem. 2011, 46, 2513-2529.

24. Ma, L.; Li, S.; Zheng, H.; Chen, J.; Lin, L.; Ye, X.; Chen, Z.; Xu, Q.; Chen, T.; Yang, J.; et al. Synthesis and biological activity of novel barbituric and thiobarbituric acid derivatives against non-alcoholic fatty liver disease. Eur. J. Med. Chem. 2011, 46, 2003-2010.

25. Zheng, H.; Li, S.; Ma, L.; Cheng, L.; Deng, C.; Chen, Z.; Xie, C.; Xiang, M.; Jiang, W.; Chen, L. A novel agonist of PPAR- $\gamma$ based on barbituric acid alleviates the development of non-alcoholic fatty liver disease by regulating adipocytokine expression and preventing insulin resistance. Eur. J. Pharmacol. 2011, 659, 244-251.

26. Gruber, P.; Rechfeld, F.; Kirchmair, J.; Hauser, N.; Boehler, M.; Garczarczyk, D.; Langer, T.; Hofmann, J. Barbituric acid derivative BAS 02104951 inhibits PKC $\varepsilon$, PKC $\eta$ PKC $/$ RACK2 interaction, Elk-1 phosphorylation in $\mathrm{HeLa}$ and $\mathrm{PKC} \varepsilon$ and $\eta$ translocation in PC3 cells following TPA-induction. J. Biochem. 2011, 149, 331-336.

27. Soine, W. Sedative-Hypnotics. In Foye's Principles of Medicinal Chemistry, 6th ed.; Foye, W.O., Lemke, T.L., Williams, D.A., Eds.; Lippincott Williams \& Wilkins: Baltimore, MD, USA, 2007; pp. 504-520.

28. Kepczyńska, E.; Obłoza, E.; Stasiewicz-Urban, A.; Bojarski, J.; Pyka, A. Lipophilicity of thiobarbiturates determined by TLC. Acta Pol. Pharm. 2007, 64, 295-302.

29. Toon, S.; Rowland, M. Structure-pharmacokinetic relationships among the barbiturates in the rat. J. Pharmacol. Exp. Ther. 1983, 225, 752-763.

30. Snider, B.B.; Patricia, J.J.; Kates, S.A. Mechanism of manganese(III)-based. Oxidation of $\beta$-keto esters. J. Org. Chem. 1988, 53, 2137-2143.

31. Citterio, A.; Sebastiano, R.; Marion, A. Synthesis of substituted tetrahydronaphthalenes by manganese(III), cerium(IV), and iron(III) oxidation of substituted diethyl alpha-benzylmalonates in the presence of olefins. J. Org. Chem. 1991, 56, 5328-5335.

32. Santi, R.; Bergamini, F.; Citterio, A.; Sebastiano, R.; Nicolini, M. Reactivity of malonyl radicals. Synthesis of substituted dihydronaphthalenes by manganese(III) oxidation of diethyl alphabenzylmalonate in the presence of alkynes. J. Org. Chem. 1992, 57, 4250-4255.

33. Bergamini, F.; Citterio, A.; Gatti, N.; Nicolini, M.; Santi, R.; Sebastiano, R. Metal-induced electrochemical oxidation of diethyl benzylmalonates in the presence of alkenes and alkynes. Synthesis of substituted tetrahydro- and dihydronaphthalenes. J. Chem. Res. (S) 1993, 9, 364-365.

34. Bouhlel, A.; Curti, C.; Vanelle, P. Access to original spirocyclic derivatives via inter- or intramolecular reaction mediated by manganese(III) acetate. Tetrahedron 2012, 68, 3596-3604.

35. Snider, B.B.; Buckman, B.O. Manganese(III) based oxidative free-radical annulations. Tetrahedron 1989, 45, 6969-6978.

36. Jagodzinska, M.; Huguenot, F.; Candiani, G.; Zanda, M. Assessing the bioisosterism of the trifluoromethyl group with a protease probe. Chem. Med. Chem. 2009, 4, 49-51. 
37. Sun, T.; Watson, S.; Manchanda, R. Disclosed are new phenobarbital salts, methods of preparation, and uses thereof. U.S. Patent 0035904, 11 February 2010; Chem. Abstr. 2010, 152, 223581.

Sample Availability: Samples of the compounds 6, 8, 10, 15, 17-24 are available from the authors.

(C) 2012 by the authors; licensee MDPI, Basel, Switzerland. This article is an open access article distributed under the terms and conditions of the Creative Commons Attribution license (http://creativecommons.org/licenses/by/3.0/). 